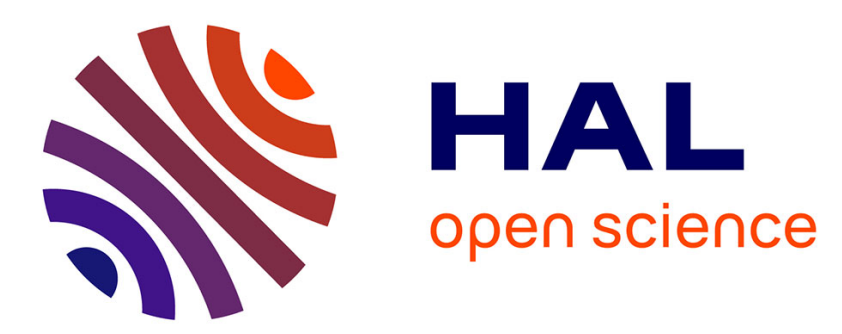

\title{
A Weak Generalized Inverse Applied to Redundancy Solving of Serial Chain Robots
}

Bertrand Tondu, Philippe Souères

\section{To cite this version:}

Bertrand Tondu, Philippe Souères. A Weak Generalized Inverse Applied to Redundancy Solving of Serial Chain Robots. International Conference on Intelligent Autonomous Systems, Jul 2014, Padova, Italy. hal-02004970

\author{
HAL Id: hal-02004970 \\ https://hal.science/hal-02004970
}

Submitted on 3 Feb 2019

HAL is a multi-disciplinary open access archive for the deposit and dissemination of scientific research documents, whether they are published or not. The documents may come from teaching and research institutions in France or abroad, or from public or private research centers.
L'archive ouverte pluridisciplinaire HAL, est destinée au dépôt et à la diffusion de documents scientifiques de niveau recherche, publiés ou non, émanant des établissements d'enseignement et de recherche français ou étrangers, des laboratoires publics ou privés. 


\title{
A Weak Generalized Inverse Applied to Redundancy Solving of Serial Chain Robots
}

\author{
Bertrand Tondu ${ }^{1,2}$ and Philippe Soueres ${ }^{2}$ \\ ${ }^{1}$ Institut National de Sciences Appliquées, University of Toulouse, 31077 Toulouse \\ ${ }^{2}$ LAAS/CNRS, 7 avenue du colonel Roche, 31400 Toulouse
}

\begin{abstract}
A peculiar form of right inverse derived from the theory of rectangular matrix determinants is considered instead of the classic Moore-Penrose psueodinverse with the aim to get compact symbolic expressions for the redundancy solving of serial chain robots. Such an approach, essentially based on the of the closed-form expressions of the $m \times m$ minors of the $m \times n$ robot Jacobian $(m<n)$, is proposed as a new way for fast computation in inverse kinematic control.
\end{abstract}

Keywords: generalized inverse, redundant robot limbs.

\section{Introduction}

Redundant robot-limbs are essentially controlled in their operational space by a numerical computation of the Moore-Penrose pseudoinverse of their Jacobian. In particular, the possibility to arbitrarily choose the associated projection operator is a powerful mean to derive benefit from the kinematic redundancy for avoiding singularities or obstacles [1], [3]. The increasing computing power of robot controllers has made possible the on-line implementation of such methods although mathematical forums sometimes emphasized the computing burden involved by the use of 'pinv' or 'pinv2'-type algorithms [4]. The goal of this paper is to analyze with a fresh eye the interest of weaker generalized inverses for deriving relatively compact symbolic expressions which could be used in some cases for a direct and simple on-line implementation.

Our start point is the classical definition of a generalized inverse of any $m \times n$ matrix $\boldsymbol{M}$ as being the matrix $\boldsymbol{X}$ satisfying at least the first or the second of the four following equations:

$$
\left\{\begin{array}{l}
\boldsymbol{M} \boldsymbol{X} \boldsymbol{M}=\boldsymbol{M} \\
\boldsymbol{X} \boldsymbol{M} \boldsymbol{X}=\boldsymbol{M} \\
(\boldsymbol{M} \boldsymbol{X})^{T}=\boldsymbol{M} \boldsymbol{X} \\
(\boldsymbol{X} \boldsymbol{M})^{T}=\boldsymbol{X} \boldsymbol{M}
\end{array}\right.
$$


The Moore-Penrose pseudoinverse is the unique matrix satisfying the four equations. Matrices satisfying only the first condition are called $\{1\}$-Inverses and they are considered as being the simplest ones [5]. We will consider in this paper a right inverse of $\boldsymbol{M}$ - i.e. a matrix $\boldsymbol{X}$ verifying $\boldsymbol{M} \boldsymbol{X}=\boldsymbol{I}_{\mathrm{m}}$ and as a consequence a $\{1,2,3\}$-Inverse - introduced in the $80^{\prime}$ by the Indian mathematician Joshi [6] in a work first devoted to determinant definition of non-rectangular matrices. In section 2, we introduce Joshi's determinant that we relate to zonotope theory and then Joshi's weak generalized inverse for which we propose an associated symbolic expression of the projection operator. In section 3, the approach is applied to the look for compact symbolic expressions of redundant robot inverse kinematic models including singularity avoidance and multiple tasks realization.

\section{Joshi's weak generalized inverse definition and its use in linear underdetermined systems solving}

Matrix determinant is a fundamental algebra notion essentially associated to square matrices. Attempts have been made to extend this notion to non-square matrices. If no global theory exists and, may be, would be meaningless in the general case of a rectangular matrix, the problem can more easily be approached if it is limited to $m \times n$ matrices with $m \leq n$. In this case, the matrix $\boldsymbol{M}$ can be read as a sequence of $n$ vectors $\left(\boldsymbol{j}_{1}, \boldsymbol{j}_{2}, \ldots, \boldsymbol{j}_{\mathrm{n}}\right)$ belonging to a $m$-dimensional vector space. An intuitive way to define a determinant associated to this vector sequence consists to consider all $m \times m$ minors by a sign, computed from their components. Radic [7] proposed at his time a first definition of the determinant of such rectangular matrices. We will however prefer a more recent definition proposed by Joshi [6] for which it is easy to show that it is equivalent to the simple form:

$$
\operatorname{det}(\boldsymbol{M})=\sum_{1 \leq i_{1}<i_{2}<\ldots<i_{m} \leq n} \operatorname{det}\left(\boldsymbol{j}_{i_{1}}, \boldsymbol{j}_{i_{2}}, \ldots, \boldsymbol{j}_{i_{m}}\right)
$$

i.e. the sum of the $\left(\begin{array}{c}n \\ m\end{array}\right) m \times m$ minors of the ordered sequence of the matrix $n$ column vectors. We will adopt this definition first due to its simplicity and also because we can geometrically justify it by means of the zonotope theory [8]. It is indeed well known that the determinant of $m$ vectors in a $m$-dimensional space can be interpreted as the oriented volume of the $m$-parallelepiped that the $m$ vectors span. In some extent, we propose to interpret the determinant of $n$ vectors in a $m$-dimensional space, as defined in Equ. (2), as the sum of the oriented volumes of the $\left(\begin{array}{c}n \\ m\end{array}\right) m$-parallelepipeds resulting from what is called a "cubical" dissection of the zonotope whose 
$\left(\boldsymbol{j}_{1}, \boldsymbol{j}_{2}, \ldots, \boldsymbol{j}_{n}\right)$ are the generators. This idea is illustrated in Fig.1: let us consider the three vectors $\left(\boldsymbol{j}_{1}, \boldsymbol{j}_{2}, \boldsymbol{j}_{3}\right)$ of the 2 -dimensional vector space; the three $2 \times 2$ minors $\operatorname{det}\left(\boldsymbol{j}_{1}, \boldsymbol{j}_{2}\right), \operatorname{det}\left(\boldsymbol{j}_{1}, \boldsymbol{j}_{3}\right)$ and $\operatorname{det}\left(\boldsymbol{j}_{2}, \boldsymbol{j}_{3}\right)$ determine three oriented areas filling the zonotope $\boldsymbol{Z}\left(\boldsymbol{j}_{1}, \boldsymbol{j}_{2}, \boldsymbol{j}_{3}\right)=\left\{\sum_{1 \leq i \leq n} \lambda_{i} \boldsymbol{j}_{i}, 0 \leq \lambda_{i} \leq 1\right\}$. From the famous Shepard-McMullen theorem [9] the volume of this zonotope is equal to $\sum_{1 \leq i_{1}<i_{2}<\ldots<i_{m} \leq n}\left|\operatorname{det}\left(\boldsymbol{j}_{i_{1}}, \boldsymbol{j}_{i_{2}}, \ldots, \boldsymbol{j}_{i_{m}}\right)\right|$. Because however minors are signed, according to the order in the vector sequence, the determinant can be equal to the zonotope volume (Fig. 1.a) or not (Fig. 1.b).

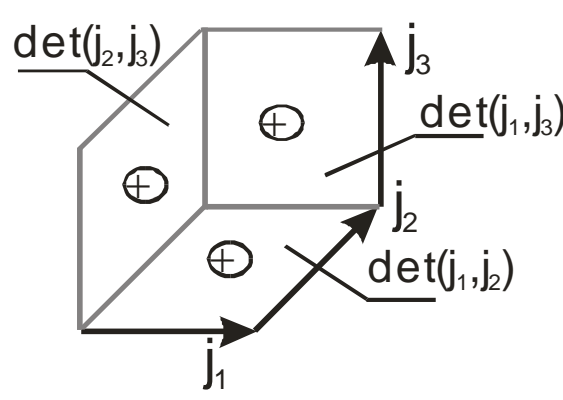

(a)

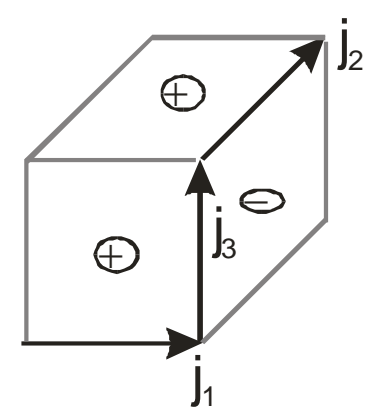

(b)

Figure 1. Geometric interpretation of the determinant definition as a sum of signed parallelepiped volumes (here parallelogram areas) resulting from a "cubical" dissection of the zonotope whose matrix column vectors $\boldsymbol{j}_{1}, \boldsymbol{j}_{2}, \boldsymbol{j}_{3}$ are the generators , (a) Case of $\operatorname{det}\left(\boldsymbol{j}_{1}, \boldsymbol{j}_{2}, \boldsymbol{j}_{3}\right)$ : all involved minors are positive and the determinant is equal to the zonotope area, (b) Case of $\operatorname{det}\left(\boldsymbol{j}_{1}, \boldsymbol{j}_{3}, \boldsymbol{j}_{2}\right)$ : two minors are positive, one is negative and the determinant is not equal to the zonotope area.

The volumic interpretation of the considered non-square matrix determinant also emphasizes a major property shared with classic determinant theory: it is independent on the base in which matrix vectors are expressed. This will be an interesting property for application to robotics. From a similar determinant definition, Joshi proposes a very appealing definition of the inverse of a rectangular $m \times n$ matrix with $m \leq n$, we will note $\boldsymbol{M}^{\mathrm{Z}}$ in reference to zonotope theory. We put:

$$
\boldsymbol{M}^{Z}=(1 / \operatorname{det}(\boldsymbol{M})) \boldsymbol{C}^{T}
$$

where $\boldsymbol{C}$ is a cofactor matrix-like, whose elements $c_{i j}, 1 \leq i \leq m, 1 \leq j \leq n$ are defined as follows: let $\boldsymbol{E}, \boldsymbol{F}, \boldsymbol{G}, \boldsymbol{H}$ be respectively the submatrices of $\boldsymbol{M}$ of the order 
$(i-1) \times(j-1), \quad(i-1) \times(n-j), \quad(m-i) \times(n-j), \quad(m-i) \times(j-1)$ such $\quad$ that

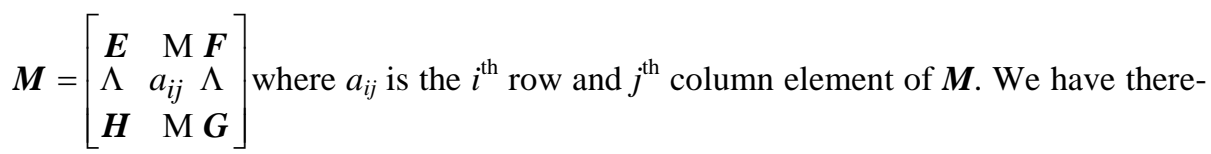
fore:

$$
\left.c_{i j}=\operatorname{det}\left[\begin{array}{cc}
\boldsymbol{E} & -\boldsymbol{F} \\
-\boldsymbol{H} & \boldsymbol{G}
\end{array}\right]\right)
$$

Let us illustrate this computation mode in the case of the Jacobian matrix $\boldsymbol{J}_{3 \mathrm{R}}$ of a classic 3R-planar robot made of three links whose lengths are respectively noted $l_{1}, l_{2}$, $l_{3}$ and associated rotation variables $\theta_{1}, \theta_{2}, \theta_{3}$; in frame $R_{0}$ shown in Fig. 2.a we get $\mathrm{S}_{i}$ and $\mathrm{C}_{i}$ respectively denote $\sin \left(\theta_{i}\right), \cos \left(\theta_{i}\right), \mathrm{S}_{i j}$ and $\mathrm{C}_{i j}, \sin \left(\theta_{i}+\theta_{j}\right)$ and $\cos \left(\theta_{i}+\theta_{j}\right)$ :

$$
J_{3 R}=\left[\begin{array}{ccc}
-l_{1} S_{1}-l_{2} S_{12}-l_{3} S_{123} & -l_{2} S_{12}-l_{3} S_{123} & -l_{3} S_{123} \\
+l_{1} C_{1}+l_{2} C_{12}+l_{3} C_{123} & +l_{2} C_{12}+l_{3} C_{123} & +l_{3} C_{123}
\end{array}\right]
$$

from which we derive the three $2 \times 2$ minors involving the three column vectors $\left(\boldsymbol{j}_{1}, \boldsymbol{j}_{2}, \boldsymbol{j}_{3}\right)$ that we will note $D_{12}, D_{13}, D_{23}$ as follows:

$$
\left\{\begin{array}{l}
D_{12}=\operatorname{det}\left(\boldsymbol{j}_{1}, \boldsymbol{j}_{2}\right)=l_{1}\left(l_{2} S_{2}+l_{3} S_{23}\right) \\
D_{13}=\operatorname{det}\left(\boldsymbol{j}_{1}, \boldsymbol{j}_{3}\right)=l_{3}\left(l_{2} S_{3}+l_{1} S_{23}\right) \\
D_{23}=\operatorname{det}\left(\boldsymbol{j}_{2}, \boldsymbol{j}_{3}\right)=l_{2} l_{3} S_{3}
\end{array}\right.
$$

and therefore the following expression of the generalized inverse results:

$$
J_{3 R}^{Z}=\frac{1}{\operatorname{det} J_{3 R}}\left[\begin{array}{cc}
l_{2} C_{12}+2 l_{3} C_{123} & l_{2} S_{12}+2 l_{3} S_{123} \\
-l_{1} C_{1}-l_{2} C_{12} & -l_{1} S_{1}-l_{2} S_{12} \\
-l_{1} C_{1}-2 l_{2} C_{12}-2 l_{3} C_{123} & -l_{1} S_{1}-2 l_{2} S_{12}-2 l_{3} S_{123}
\end{array}\right]
$$

where: $\operatorname{det} J_{3 R}=D_{12}+D_{13}+D_{23}=l_{1} l_{2} S_{2}+2 l_{2} l_{3} S_{3}+2 l_{1} l_{3} S_{23}$.

It is particularly interesting to note that the matrix of "cofactors" has a complexity in terms of algebraic or sinus/cosinus operations similar to this of the Jacobian matrix from which it results, with an associated relatively compact determinant expression. Although, in the case of this example, $\left(J_{3 R} J_{3 R}^{T}\right)$ is a $2 \times 2$ matrix, the components of the closed-form of the Moore-Penrose pseudoinverse $\boldsymbol{J}_{3 R}^{+}=\boldsymbol{J}_{3 R}^{T}\left(\boldsymbol{J}_{3 R} \boldsymbol{J}_{3 R}^{T}\right)^{-1}$ are much more complex to express, even after all trigonometric simplifications were made. The symbolic compactness of Joshi's inverse of any $m \times n$ matrix $\boldsymbol{M}=\left(\boldsymbol{j}_{1}, \boldsymbol{j}_{2}, \ldots, \boldsymbol{j}_{n}\right)$ however has a major drawback: while the pseudoinverse becomes 
singular if and only if $\sum_{1 \leq i_{1}<i_{2}<\ldots<i_{m} \leq n} \operatorname{det}^{2}\left(\boldsymbol{j}_{i_{1}}, \boldsymbol{j}_{i_{2}}, \ldots, \boldsymbol{j}_{i_{m}}\right)=0$, Joshi's inverse becomes singular if and only if $\sum_{1 \leq i_{1}<i_{2}<\ldots<i_{m} \leq n} \operatorname{det}\left(\boldsymbol{j}_{i_{1}}, \boldsymbol{j}_{i_{2}}, \ldots, \boldsymbol{j}_{i_{m}}\right)=0$. In other words, by comparison with Moore-Penrose pseudoinverse, Joshi's inverse introduces the algorithmic singularities corresponding to a sum of considered minors equal to zero without any single minor is equal to zero. We will see however in next paragraph how to avoid any singularity.

Let us consider now the underdetermined linear equation $\boldsymbol{v}=\boldsymbol{M u}$ where $\boldsymbol{v}$ is a $m$ dimensional vector and $\boldsymbol{u}$ a $n$-dimensional vector $(m<n)$. Because Joshi's inverse is a $\{1,2,3\}$-Inverse of $\boldsymbol{M}$, the general solution of the linear equation is given, if $\operatorname{det}(\boldsymbol{M}) \neq 0$, by:

$$
\boldsymbol{u}=\boldsymbol{M}^{Z} \boldsymbol{v}+\left(\boldsymbol{I}_{n}-\boldsymbol{M}^{Z} \boldsymbol{M}\right) \boldsymbol{z}
$$

where $z$ is an arbitrary $n \times 1$ matrix. According to our look for compact symbolic expressions the question is: what is the compactness of the projection operator $\left(\boldsymbol{I}_{\mathrm{n}}-\boldsymbol{M}^{\boldsymbol{Z}} \boldsymbol{M}\right)$ ? We first want to emphasize a point shared by any right inverse and therefore also by the Moore-Penrose inverse: $\left(\boldsymbol{I}_{\mathrm{n}}-\operatorname{Inverse}(\boldsymbol{M}) \boldsymbol{M}\right)$ is independent on the base in which column vectors of $\boldsymbol{M}$ are expressed. Let us consider $\boldsymbol{M}=\boldsymbol{M}_{1} \boldsymbol{M}_{2}$ where $\boldsymbol{M}_{2}$ is a $m \times n$ matrix $(m<n)$ and $\boldsymbol{M}_{1}$ is a square $m \times m$ matrix, $\boldsymbol{u}=\boldsymbol{M}^{Z} \boldsymbol{v}+\left(\boldsymbol{I}_{n}-\boldsymbol{M}_{2}^{Z} \boldsymbol{M}_{2}\right) \boldsymbol{z}$ is a general solution of $\boldsymbol{v}=\boldsymbol{M u}$ since: $\boldsymbol{M u}=\boldsymbol{M} \boldsymbol{M}^{Z} \boldsymbol{v}+\left(\boldsymbol{M}-\boldsymbol{M}_{1} \boldsymbol{M}_{2} \boldsymbol{M}_{2}^{Z} \boldsymbol{M}_{2}\right) \boldsymbol{z}=\boldsymbol{v}$. Let us consider a reference 0 -base in which are expressed the column vectors of the matrix ${ }^{0} \boldsymbol{M}$; it follows that the general solution ${ }^{0} \boldsymbol{v}={ }^{0} \boldsymbol{M} \boldsymbol{u}$ is given, if $\operatorname{det}(\boldsymbol{M}) \neq 0$, by:

$$
\boldsymbol{u}={ }^{0} \boldsymbol{M}^{Z 0} \boldsymbol{v}+\left(\boldsymbol{I}_{n}-{ }^{k} \boldsymbol{M}^{Z k} \boldsymbol{M}\right) \boldsymbol{z}
$$

where $z$ is an arbitrary $n \times 1$ matrix and ' $k$ ' designates an arbitrary base of the considered $m$-dimensional space. In what follows, the base in which the projector is expressed will be omitted. This result suggests that $\left(\boldsymbol{I}_{\mathrm{n}}-\boldsymbol{M}^{\boldsymbol{Z}} \boldsymbol{M}\right)$ could be expressed only with minors since these last ones are base-independent. In the case where $m=2$ and any $n$, we get, for $\boldsymbol{M}=\left[a_{i j}\right]$ with $i=1$ to 2 and $j=1$ to $n$, and $\boldsymbol{C}$ its cofactor matrix:

$$
\boldsymbol{C}^{T} \boldsymbol{M}=\left[\begin{array}{cc}
+a_{22}+a_{23}+\ldots+a_{2 n} & -a_{12}-a_{13}-\ldots-a_{1 n} \\
-a_{21}+a_{23}+\ldots+a_{2 n} & +a_{11}-a_{13}-\ldots-a_{1 n} \\
\mathrm{M} & \mathrm{M} \\
-a_{21}-a_{22}-\ldots-a_{2 n-1} & +a_{11}+a_{12}+\ldots+a_{1 n-1}
\end{array}\right]\left[\begin{array}{llll}
a_{11} & a_{12} & \ldots & a_{1 n} \\
a_{21} & a_{22} & \ldots & a_{2 n}
\end{array}\right]
$$

whose all resulting terms clearly correspond to a sum of minors $D_{k j}=\left(a_{1 k} a_{2 j}-a_{1 j} a_{2 k}\right)$, $k<j$, by a sign. A similar result can also be simply deduced in the case where $m=3$ and any $n$. This suggests the following conjecture we however not tried to demonstrate in the general case to privilege the robotic point of view: 


$$
\operatorname{det}(\boldsymbol{M}) \boldsymbol{M}^{Z} \boldsymbol{M}=\left[\sum_{\substack{1 \leq k_{2}<k_{3}<\ldots<k_{m} \leq n \\ k_{2}, k_{3}, \ldots, k_{m} \notin\{i, j\}}} \sigma_{i k_{2} k_{3} . k_{m}} D_{j k_{2} k_{3} \ldots k_{m}}\right]_{\substack{1 \leq i \leq n, 1 \leq \mathrm{j} \leq \mathrm{n}\\}}
$$

where $\sigma_{i k_{2} k_{3} \ldots k_{m}}$ is equal to 1 if $i<k_{2}$ and -1 if not, and $D_{j k_{2} k_{3} . . k_{m}}=\operatorname{det}\left(\boldsymbol{j}_{j}, \boldsymbol{j}_{k_{2}}, \ldots, \boldsymbol{j}_{k_{m}}\right)$. In the case of our planar 3R-robot, we get:

$$
\begin{gathered}
\operatorname{det}\left(\boldsymbol{J}_{3 R}\right) \boldsymbol{J}_{3 R}{ }^{Z} \boldsymbol{J}_{3 R}=\left[\begin{array}{ccc}
D_{12}+D_{13} & D_{23} & -D_{23} \\
D_{13} & D_{12}+D_{23} & D_{13} \\
-D_{12} & D_{12} & D_{13}+D_{23}
\end{array}\right] \\
\Rightarrow\left(\boldsymbol{I}_{3}-\boldsymbol{J}_{3 R}{ }^{Z} \boldsymbol{J}_{3 R}\right)=\frac{1}{D_{12}+D_{13}+D_{23}}\left[\begin{array}{ccc}
D_{23} & -D_{23} & D_{23} \\
-D_{13} & D_{13} & -D_{13} \\
D_{12} & -D_{12} & D_{12}
\end{array}\right]
\end{gathered}
$$

The claimed result of Equ. (11) opens the way to the development of original algorithms for solving underdetermined problems based on the computation of the matrix $m \times m$ minors in some alternative way to the classic singular value decomposition applied to the Moore-Penrose inverse. Moreover, it is easy to show that the number of minors involved in the computation of each term of the matrix $\operatorname{det}(\boldsymbol{M})\left(\boldsymbol{I}_{n}-\boldsymbol{M}^{Z} \boldsymbol{M}\right)$ is equal to $\left(\begin{array}{c}n \\ m\end{array}\right)-\left(\begin{array}{c}n-1 \\ m-1\end{array}\right)$ on the diagonal and to $\left(\begin{array}{c}n-2 \\ m-1\end{array}\right)$ outside. Consequently, very compact symbolic expressions can be hoped for a low degree $r$ of redundancy defined as being $r=(n-m)$, especially for $r=1$ or $r=2$, which concerns most of redundant industrial robots; in particular, if $r=1$, each term of the projection operator matrix is equal to one single minor.

\section{Application to redundancy solving of serial chain redundant robots}

The matrix $\boldsymbol{M}$ is now the $m \times n$ Jacobian matrix denoted $\boldsymbol{J}$ of a serial chain robot with a number $n$ of degrees of freedom greater than necessary to perform a given task in a $m$-dimensional operational space. The joint vector will be noted $\boldsymbol{q}$ and its components $q_{i}, 1 \leq i \leq n$ while the operational vector will be noted $\boldsymbol{x}$. Since historical works by Nakamura [1] redundancy solving, in a kinematic point of view, can take two forms: the optimization of a criterion on the one hand, the realization of multiple tasks on the other hand.

\subsection{Criterion optimization}

In the case of the pseudoinverse, the arbitrarily vector $z$ associated to the projection operator $\left(\boldsymbol{I}_{\mathrm{n}}-\boldsymbol{J}^{Z} \boldsymbol{J}\right)$ can be used for maximizing a criterion thanks to the fact that 
$\left(\boldsymbol{I}_{\mathrm{n}}-\boldsymbol{J}^{Z} \boldsymbol{J}\right)$ is a nonnegative definite matrix. That is not the case for a $\{1,2,3\}$-Inverse but this difficulty can be simply overcome if we define $z$ as follows:

$$
\boldsymbol{z}=k_{c}\left(\boldsymbol{I}_{n}-\boldsymbol{J}^{Z} \boldsymbol{J}\right)^{T}\left[\frac{\partial \operatorname{Crit}(\boldsymbol{q})}{\partial q_{1}} \frac{\partial \operatorname{Crit}(\boldsymbol{q})}{\partial q_{2}} \ldots \frac{\partial \operatorname{Crit}(\boldsymbol{q})}{\partial q_{n}}\right]^{T}
$$

where $\operatorname{Crit}(\boldsymbol{q})$ is a criterion to be maximized by the robot and $k_{c}$ a positive real. By comparison with Moore-Penrose pseudoinverse computation, the supplementary term $\left(\boldsymbol{I}_{\mathrm{n}}-\boldsymbol{J}^{Z} \boldsymbol{J}\right)^{T}$ has a negative effect on the look for a final compact symbolic expression of \&but we think that this effect is limited by the symbolic form $\left(\boldsymbol{I}_{\mathrm{n}}-\boldsymbol{J}^{\boldsymbol{Z}} \boldsymbol{J}\right)$ which can be derived from Equ. (11) especially for low redundancy degree, as we are going to illustrate it. Moreover, in the practical important case of the look for singularity avoidance, and because our definition of the matrix determinant includes the robot singularities, we can consider, instead of the manipulability criterion, the following simpler one:

$$
\operatorname{Crit}(\boldsymbol{q})=\operatorname{det}^{2}(\boldsymbol{J}) \Rightarrow\left[\frac{\partial \operatorname{Crit}(\boldsymbol{q})}{\partial q_{i}}\right]_{1 \leq i \leq n}^{T}=2 \operatorname{det}(\boldsymbol{J})\left[\frac{\partial \operatorname{det}(\boldsymbol{J})}{\partial q_{i}}\right]_{1 \leq i \leq n}^{T}
$$

where the terms $\left(\frac{\partial \operatorname{det}(\boldsymbol{J})}{\partial q_{i}}\right)$ are easy to deduce in closed form from the knowledge of minors expressions. Let consider for example the case of a $3 \mathrm{R}$ planar robot whose Jacobian inverse matrix $J_{3 R}^{Z}$ is given in Equ. (7). From the symbolic expression of its first minors $D_{12}, D_{13}$ and $D_{23}$ given in Equ. (6) and from Equ. (11), after all simplifications were made, we finally get the final expression of \&including the satisfaction of singularity avoidance by means of the positive real $k_{c}-$ in fact it would be $6 k_{c}$ from Equ. (13):

$$
\&=J_{3 R}^{Z} \mathrm{\&}+k_{c}\left(2 l_{3}\left(l_{2} C_{3}+l_{1} C_{23}\right) M_{12}-M_{13} l_{1}\left(l_{2} C_{2}+2 l_{3} C_{23}\right)\right)\left[\begin{array}{l}
+M_{23} \\
-M_{13} \\
+M_{12}
\end{array}\right]
$$

where $C_{2}, C_{3}$ and $\mathrm{C}_{23}$ respectively denote $\cos \left(\theta_{2}\right), \cos \left(\theta_{3}\right)$ and $\cos \left(\theta_{2}+\theta_{3}\right)$. We applied this equation to the example proposed by Yoshikawa in his book [10] (page 255): the planar 3R-robot with link lengths equal to $l_{1}=l_{2}=1, l_{3}=0.3$ must perform a straight-line path from the initial configuration $\boldsymbol{q}_{0}=\left[180^{\circ},-170^{\circ},-10^{\circ}\right]^{\mathrm{T}}$, corresponding to the initial operational end-position $\left[P_{\mathrm{x} 0}, P_{\mathrm{y} 0}\right]^{\mathrm{T}} \approx[0.28,0.17]^{\mathrm{T}}$ with respect to frame $\left(O_{0}\right.$, $\left.X_{0}, Y_{0}\right)$, to the final one $\left[P_{\mathrm{x} 0},-0.1\right]^{\mathrm{T}}$ according to the desired trajectory $\boldsymbol{x}(t)=\left[P_{x 0}, P_{y 0}-(3-2 t) t^{2}\left(P_{y 0}+0.1\right)\right]^{\top}, 0 \leq t \leq 1$ (see Fig. 2.a). We give in Fig. 2.b and 2.d the comparison between our approach and the classic pseudoinverse approach with maximization of the robot manipulability as defined by Yoshikawa $\sqrt{\operatorname{det}\left(\boldsymbol{J}_{3 R} \boldsymbol{J}_{3 R}^{T}\right)}$. It clearly appears that our approach can lead to a result very close to 
this given by the pseudoinverse with very close changes for the manipulability criterion (see Fig. 2.c).

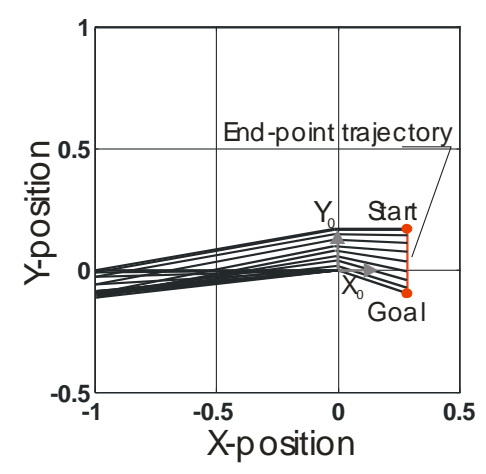

(a)

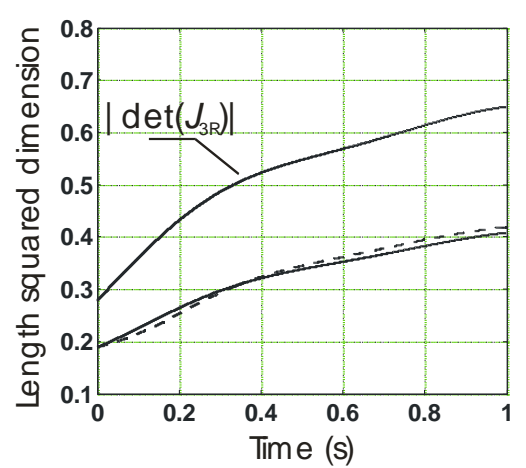

(c)

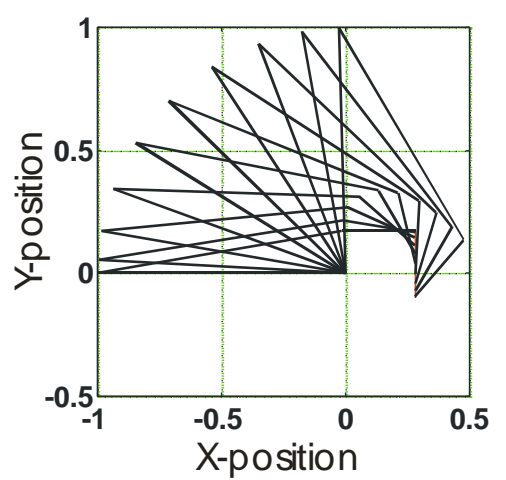

(b)

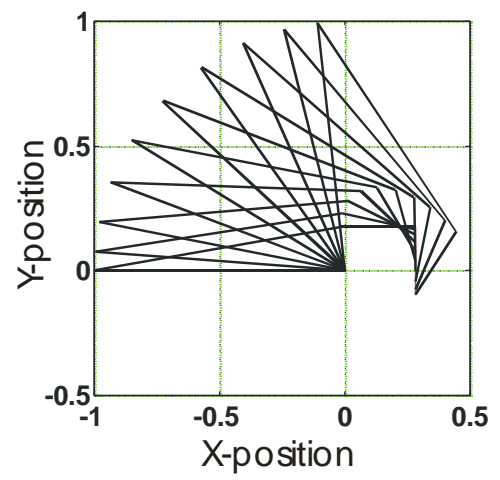

(d)

Figure 2. Simulation of a 3R-planar robot whose redundancy is used to avoid singularity in the Cartesian plane while its end-point must perform a straight-line path with some imposed velocity profile, (a) Pseudoinverse without criterion, (b) Pseudoinverse with criterion, (c) Manipulability comparison between pseudoinverse with criterion (continuous line) and proposed method with criterion (dotted line), (d) Proposed method with criterion $\left(k_{\mathrm{c}}=35\right)$.

\subsection{Multiple tasks}

In the case of the redundancy use for performing multiple tasks, the following wellknown formula was established by Nakamura [1]:

$$
\&=\boldsymbol{J}_{1}^{+} \&+\tilde{\boldsymbol{J}}_{2}^{+}\left(\varkappa_{2}-\tilde{\boldsymbol{J}}_{2} \boldsymbol{J}_{1}^{+} \&\right)+\left(\boldsymbol{I}_{n}-\boldsymbol{J}_{1}^{+} \boldsymbol{J}_{1}\right)\left(\boldsymbol{I}_{n}-\tilde{\boldsymbol{J}}_{2}^{+} \tilde{\boldsymbol{J}}_{2}\right) \boldsymbol{z}
$$


where $\mathcal{A}_{1}=\boldsymbol{J}_{1} \&$ defines the first task with $\boldsymbol{J}_{1}$ a $m \times n$ matrix $(m<n), \boldsymbol{J}_{2} \propto \boldsymbol{J}_{2}$ defines the second task with $\boldsymbol{J}_{2}$ a $p \times n$ matrix $(p<m), \tilde{\boldsymbol{J}}_{2}=\boldsymbol{J}_{2}\left(\boldsymbol{I}_{n}-\boldsymbol{J}_{1}^{+} \boldsymbol{J}_{1}\right)$ and $z$ an arbitrary $n \times 1$ vector. The same approach applied to our $\{1,2,3\}$-Inverse leads to:

$$
\boldsymbol{q}=\boldsymbol{J}_{1}^{Z} \&+\left(\boldsymbol{I}_{n}-\boldsymbol{J}_{1}^{Z} \boldsymbol{J}_{1}\right) \tilde{\boldsymbol{J}}_{2}^{Z}\left({ }_{2}-\tilde{\boldsymbol{J}}_{2} \boldsymbol{J}_{1}^{Z} \&\right)+\left(\boldsymbol{I}_{n}-\boldsymbol{J}_{1}^{Z} \boldsymbol{J}_{1}\right)\left(\boldsymbol{I}_{n}-\tilde{\boldsymbol{J}}_{2}^{Z} \tilde{\boldsymbol{J}}_{2}\right) \boldsymbol{z}
$$

where $\tilde{\boldsymbol{J}}_{2}=\boldsymbol{J}_{2}\left(\boldsymbol{I}_{n}-\boldsymbol{J}_{1}^{Z} \boldsymbol{J}_{1}\right)$ and $z$ an arbitrary $n \times 1$ vector. But, by comparison with the $\{1,2,3,4\}$-Inverse, we can not write ' $\left(\boldsymbol{I}_{n}-\boldsymbol{J}_{1}^{Z} \boldsymbol{J}_{1}\right) \tilde{\boldsymbol{J}}_{2}^{Z}=\tilde{\boldsymbol{J}}_{2}^{Z}$ '. However the expected advantage of Equ. (17) by comparison with Equ. (16) is to derive benefit from compact symbolic expressions of $\boldsymbol{J}_{1}^{Z}, \tilde{\boldsymbol{J}}_{2}^{Z},\left(\boldsymbol{I}_{n}-\boldsymbol{J}_{1}^{Z} \boldsymbol{J}_{1}\right)$ as from $\left(\boldsymbol{I}_{n}-\tilde{\boldsymbol{J}}_{2}^{Z} \tilde{\boldsymbol{J}}_{2}\right)$ in order to get a final symbolic expression of \&expression able to favour a fast computation of the redundancy solving. Let us illustrate this approach in the case of a 4Rplanar robot with a degree of redundancy equal to 2 i.e. $n=4$ and $m=2$ : the first task consists in positioning the robot end-point in the Cartesian plane. From an obvious extension of $\boldsymbol{J}_{3 \mathrm{R}}$-matrix expression given in Equ. (5) for defining $\boldsymbol{J}_{4 \mathrm{R}}$, we easily derive the following expression of $J_{4 R}^{Z}$ - as previously the successive link-lengths of the robot are denoted $l_{\mathrm{i}}$ and its joint variables $\theta_{\mathrm{i}}$, for $i=1$ to 4 :

$$
\boldsymbol{J}_{4 R}^{Z}=\frac{1}{\operatorname{det}\left(\boldsymbol{J}_{4 R}\right)}\left[\begin{array}{cc}
l_{2} C_{12}+2 l_{3} C_{123}+3 l_{4} C_{1234} & -l_{2} S_{12}-2 l_{3} S_{123}-3 l_{4} S_{1234} \\
-l_{1} C_{1}-l_{2} C_{12}+3 l_{4} C_{1234} & -l_{1} S_{1}-l_{2} S_{12}+l_{4} S_{1234} \\
-l_{1} C_{1}-2 l_{2} C_{12}-2 l_{3} C_{123}-l_{4} C_{1234} & -l_{1} S_{1}-2 l_{2} S_{12}-2 l_{3} S_{123}-l_{4} S_{1234} \\
-l_{1} C_{1}-2 l_{2} C_{12}-3 l_{3} C_{123}-3 l_{4} C_{1234} & -l_{1} S_{1}-2 l_{2} S_{12}-3 l_{3} S_{123}-3 l_{4} S_{1234}
\end{array}\right]
$$

The determinant of $\boldsymbol{J}_{4 \mathrm{R}}$ is the sum of the six minors $D_{i_{1} i_{2}}=\operatorname{det}\left(\boldsymbol{j}_{i_{1}}, \boldsymbol{j}_{i_{2}}\right)$ for $1 \leq i_{1}<i_{2} \leq 4$ where $\boldsymbol{j}_{\mathrm{k}}$ is the $k^{\text {th }}$ column-vector of $\boldsymbol{J}_{4 \mathrm{R}}$ for $k=1$ to 4 ; we get:

$$
\begin{cases}D_{12}=l_{1}\left(l_{2} S_{2}+l_{3} S_{23}+l_{4} S_{234}\right) ; & D_{13}=l_{3}\left(l_{2} S_{3}+l_{1} S_{23}\right)+l_{4}\left(l_{2} S_{34}+l_{1} S_{234}\right) \\ D_{14}=l_{4}\left(l_{3} S_{4}+l_{2} S_{34}+l_{1} S_{234}\right) ; & D_{23}=l_{2}\left(l_{3} S_{3}+l_{4} S_{34}\right) \\ D_{24}=l_{4}\left(l_{3} S_{4}+l_{2} S_{34}\right) ; & D_{34}=l_{3} l_{4} S_{4}\end{cases}
$$

and therefore:

$$
\operatorname{det} J_{1}=l_{1} l_{2} S_{2}+2 l_{2} l_{3} S_{3}+2 l_{3} l_{4} S_{4}+2 l_{1} l_{3} S_{23}+4 l_{2} l_{4} S_{34}+3 l_{1} l_{4} S_{234}
$$

The second task consists in using the two degrees of freedom brought by the redundancy to avoid the obstacle shown in Fig. 3.a by imposing adapted joint 3 and joint 4 trajectories denoted respectively $\otimes_{3 d}^{\&}$ and $\&_{4 d}^{\&}$. We get, as a consequence:

$$
\boldsymbol{J}_{2}=\left[\begin{array}{llll}
0 & 0 & 1 & 0 \\
0 & 0 & 0 & 1
\end{array}\right]
$$


We will now note $\boldsymbol{J}_{4 \mathrm{R}}=\boldsymbol{J}_{1}$. From Equ. (11) we derive:

$$
\begin{aligned}
& \operatorname{det}\left(\boldsymbol{J}_{1}\right)\left(\boldsymbol{I}_{4}-\boldsymbol{J}_{1}^{Z} \boldsymbol{J}_{1}\right)= \\
& {\left[\begin{array}{cccc}
D_{23}+D_{24}+D_{34} & -D_{23}-D_{24} & D_{23}-D_{34} & D_{24}+D_{34} \\
-D_{13}-D_{14} & D_{13}+D_{14}+D_{34} & -D_{13}-D_{34} & -D_{14}+D_{34} \\
D_{12}-D_{14} & -D_{12}-D_{24} & D_{12}+D_{14}+D_{24} & -D_{14}-D_{24} \\
D_{12}+D_{13} & -D_{12}+D_{23} & -D_{13}-D_{23} & D_{12}+D_{13}+D_{23}
\end{array}\right]}
\end{aligned}
$$

from which we can easily get symbolic expressions of $\tilde{\boldsymbol{J}}_{2}$ and then $\left(\boldsymbol{I}_{4}-\boldsymbol{J}_{1}^{Z} \boldsymbol{J}_{1}\right) \tilde{\boldsymbol{J}}_{2}^{Z}$.

Finally we check that $\frac{\&}{3}=\frac{\& d}{3}, \&=\& 4$ and we get:

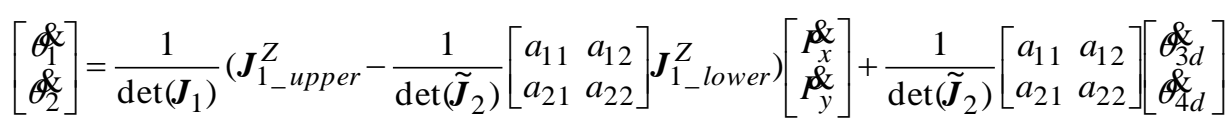

where $\boldsymbol{J}_{1 \_ \text {upper }}^{Z}, \boldsymbol{J}_{1 \_ \text {lower }}^{Z}$ are the $2 \times 2$ upper and lower sub-matrices of $\operatorname{det}\left(\boldsymbol{J}_{1}\right) \boldsymbol{J}_{1}^{Z}$, as expressed in Equ. 18 and:

$$
\left\{\begin{array}{c}
a_{11}=+D_{23}\left(\operatorname{det}\left(\boldsymbol{J}_{1}\right)-D_{14}\right)-D_{12} D_{34}+D_{13} D_{24} \\
a_{12}=+D_{24}\left(\operatorname{det}\left(\boldsymbol{J}_{1}\right)-D_{13}\right)+D_{12} D_{34}+D_{14} D_{23} \\
a_{21}=-D_{13}\left(\operatorname{det}\left(\boldsymbol{J}_{1}\right)-D_{24}\right)-D_{12} D_{34}-D_{14} D_{23} \\
a_{22}=-D_{14}\left(\operatorname{det}\left(\boldsymbol{J}_{1}\right)-D_{34}\right)+D_{12} D_{34}-D_{13} D_{24} \\
\operatorname{det}\left(\widetilde{\boldsymbol{J}}_{2}\right)=D_{12}\left(\operatorname{det}\left(\boldsymbol{J}_{1}\right)-D_{34}\right)+D_{13} D_{24}-D_{14} D_{23}
\end{array}\right.
$$

Moreover it is easy to show that $\left(\boldsymbol{I}_{n}-\boldsymbol{J}_{1}^{+} \boldsymbol{J}_{1}\right)\left(\boldsymbol{I}_{n}-\tilde{\boldsymbol{J}}_{2}^{+} \tilde{\boldsymbol{J}}_{2}\right)=\boldsymbol{O}_{4}$ which means that all the redundancy capability is here used for avoiding the obstacle. The symbolic expressions involved in the algorithm are now more complex than in the case of the previous 3R-planar robot example but they are still acceptable for an on-line computation.

We show in Fig. 3 the simulation of the algorithm on the following example: the 4Rplanar robot whose all links have a length equal to 0.25 is initially set in a start configuration $\boldsymbol{q}_{0}=\left[45^{\circ},-10^{\circ},-20^{\circ}, 30^{\circ}\right]^{\mathrm{T}}$ from which it must move in a straight-line according to a trapezoidal speed profile - constant cruising speed is equal to 1 (link length dimension)/s and constant initial/final accelerations are equal to 2 (link length dimension) $/ \mathrm{s}^{2}$ ) - to a final position with same $X$-value and a $Y$-value equal to zero. In order to avoid the obstacle, the trajectories of $\theta_{3}$ and $\theta_{4}$ are imposed also according to a given trapezoidal speed profile from their following initial positions to the final one $\left(\theta_{3 f}, \theta_{4 f}\right)=\left(-25^{\circ},-45^{\circ}\right)$. If this second task is not specified, the robot end-point follows the straight-line imposed by the first task but comes against the obstacle (Fig. 3.b). Thanks to the second task, the obstacle is avoided (Fig. 3.c) with joint trajectories in $\theta_{1}$ and $\theta_{2}$ deprived of excessive slopes as shown in Fig. 3.d. 


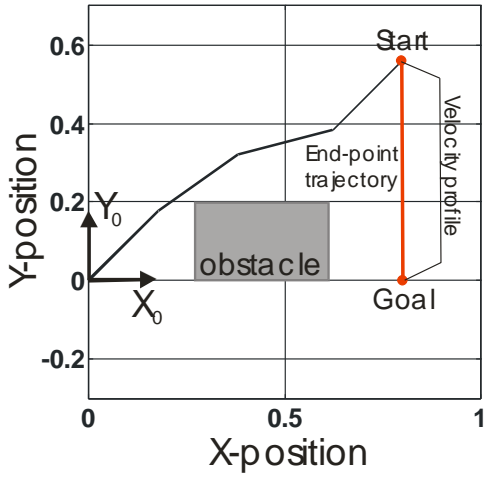

(a)

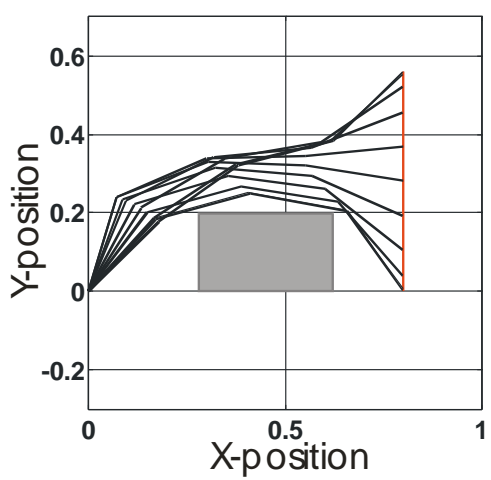

(c)

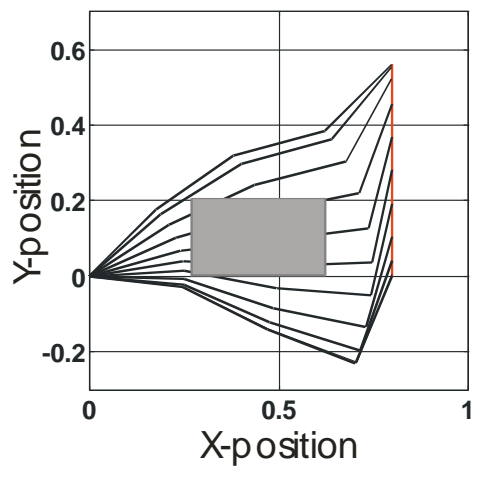

(b)

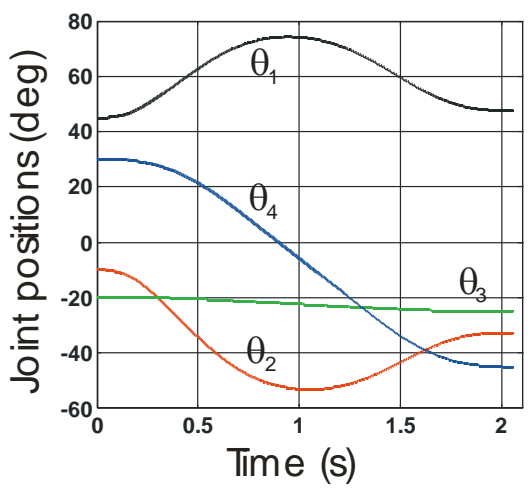

(d)

Figure 3. Simulation of a 4R-planar robot whose redundancy is used for following a straightline path (first task) while avoiding an obstacle thanks to predefined joint trajectories in $\theta_{3}$ and $\theta_{4}$ (second task), (a) Initial configuration and location of the obstacle, (b) Redundancy solving without the second task, (c) Redundancy solving with the second task, (d) Corresponding joint variables to the performance of both first and second tasks.

\section{Conclusion}

We proposed a symbolic approach for a fast computation of redundancy solving including optimization criterion and multiple tasks realization. Our approach is founded on the determinant definition of a $m \times n$ rectangular matrix $(m<n)$ as the sum of its $\left(\begin{array}{c}n \\ m\end{array}\right) m \times m$ ordered minors, and the right inverse which can be associated to it. We highlighted the fact that each term of the projection operator associated to this inverse can be written as a limited sum of these minors, leading to a general redundancy solving algorithm based on the symbolic expressions of the robot Jacobian minors. The 
proposed method however suffers the combinatorial increasing of the number of considered minors, especially when the robot is considered in the 6-dimensional operational space with a high degree of redundancy. We think however that the method can be particularly efficient in some important cases: on the one hand, in the plane, for $n \mathrm{R}$ snake-like robots, even with a high $n$-number of degrees of freedom, due to the fact we can hope to get recursive equations for the minor expressions as this is suggested by the obtained expressions in $3 \mathrm{R}$ and $4 \mathrm{R}$-planar robot cases; on the other hand, for any spatial robot whose degree of redundancy is equal to 1 , as it is the case for the classic 7R-arm, involving therefore seven $6 \times 6$ minors and a projection operator matrix $\operatorname{det}(\boldsymbol{J})\left(\boldsymbol{I}_{n}-\boldsymbol{J}^{Z} \boldsymbol{J}\right)$ whose each term is equal to only one minor.

\section{References}

1. Y. Nakamura, Advanced Robotics, Redundancy and Optimization, Addison-Wesley, Longman Publishing, 1991.

2. S. Chiaverini, "Singularity-Robust Task-Priority Redundancy Resolution for Real-Time Kinematic Control of Robust Manipulators", IEEE Trans. on Robotics and Automation, Vol. 13, N³, June 1997, pp. 398-410.

3. Y. Choi, Y. Oh, S.R. Oh, J. Park and W.K. Chung, "Multiple Tasks Manipulation for a Robotic Manipulator”, Advanced Robotics, Vol. 18, N6, 2004, pp. 637-653.

4. Vene, "My Programming and Machine Learning Blog: Inverses, Pseudoinverses. Numerical Issues, Speed, Symmetry", August $18^{\text {th }} 2012$, http://blog.vene.ro.

5. V. Lovass-Nagy, R.J. Miller and D.L. Powers, "An Introduction to the Application of the Simplest Matrix-Generalized Inverse in Systems Science", IEEE Trans. on Circuits and Systems, Vol. CAS-25, $\mathrm{N}^{\circ}$ 9, Sept. 78, pp. 766-771.

6. V.N. Joshi, "A determinant for rectangular matrices", Bulletin of Australian Mathematical Society, Vol. 21, 1980, pp. 137-146.

7. M. Radic, "A definition of the determinant of a rectangular matrix", Glasnik Matematicki, Vol. 1, N¹, 1966, pp. 17-21.

8. P. McMullen, "On zonotopes", Transactions of the American Mathematical Society, Vol. 159, 1971, pp. 91-109.

9. G.C. Shephard, "Combinatorial properties of associated zonotopes", Canadian Journal of Mathematics, Vol. XXVI, N², 1974, pp. 302-321.

10. T. Yoshikawa, Foundations of Robotics, Analysis and Control, The MIT Press; Cambridge, Massachusetts, 1990. 